\title{
Evaluation of SARS-CoV-2 Existence in Blood, Urine, and Rectal Swab in Positive Patients with Different Virus Titers
}

\author{
Mahdieh Mondanizadeh ${ }^{1}$, Ensiye Hrahimi ${ }^{2}$, Hossein Sarmadian ${ }^{2}$, Mohammad Jamalian ${ }^{3}$ and \\ Behzad Khansarinejad (ii) ${ }^{3, *}$ \\ ${ }^{1}$ Department of Biotechnology and Molecular Medicine, Arak University of Medical Sciences, Arak, Iran \\ ${ }^{2}$ Department of Infectious Disease, School of Medicine, Arak University of Medical Sciences, Arak, Iran \\ ${ }^{3}$ Department of Microbiology and Immunology, Arak University of Medical Sciences, Arak, Iran \\ "Corresponding author: Associate Professor, Department of Microbiology and Immunology, Arak University of Medical Sciences, Arak, Iran. Tel: +988634172526, Email: \\ b_khansarinejad@yahoo.com
}

Received 2020 June 16; Revised 2020 October 06; Accepted 2020 November 06.

\begin{abstract}
Background: Coronavirus disease 2019 (COVID-19) is an infectious disease caused by a newly discovered coronavirus. Understanding the spreading routes of SARS-CoV-2 is crucial for patient management and defining biosafety strategies for public and health care workers.

Objectives: In the current study, the virus shedding in the upper respiratory as well as blood, stool, and urine specimens of infected patients, was examined using quantitative real-time PCR assay (qRT-PCR).

Methods: The samples of 50 positive patients with high, moderate, and low virus titers in their respiratory specimens were collected. All specimens were subjected to RNA extraction and RT-qPCR assay using two different internally-controlled test systems. Results: The results indicated that no positive results were observed in the urine samples of all patients. The viral genome was diagnosed in $5 \%$ of blood and 3.3\% of rectal swab samples. The Cq (Cycle of quantification)-values of positive results on the stool and blood samples were always higher than the Cq-value of the respiratory specimen of the same patient.

Conclusions: SARS-CoV-2 is mainly detected in the respiratory samples, and the virus is not detectable in the urine. The importance of viremia and the existence of the virus in feces in virus spread in the human population needs further investigation.
\end{abstract}

Keywords: COVID-19, Coronavirus, SARS-CoV-2, RT-qPCR

\section{Background}

Since the emergence and spread of severe acute respiratory syndrome coronavirus 2 (SARS-CoV-2) in late 2019 from Wuhan (Hubei province, China), several countries have reported sporadic imported cases among travelers returning from China $(1,2)$. The disease became a global concern and is now spreading rapidly across the globe. The symptoms of the disease include fever, dry cough, fatigue, myalgia, and dyspnea. In addition, some patients might suffer from headaches, dizziness, abdominal pain, diarrhea, nausea, and vomiting (3). The onset of the disease may lead to progressive respiratory failure due to alveolar damage and even death (4). On April 7, 2020, a total of laboratory-confirmed 1,348,628 cases had been reported in at least 184 countries, causing more than 74,834 deaths (5).

In Iran, the first cases of the virus were diagnosed on 18
February in the respiratory samples of two patients from Qom city. The third case was diagnosed on the same date in Arak city by the specialized virology laboratory of Imam Reza clinic (Arak, Iran). Until April 7, a total number of 60,500 laboratory-confirmed cases and 3,872 deaths were reported from the country. The spreading routes of the virus have been a great concern, both for public and health care workers. Answering this question is essential for patient management and defining biosafety strategies.

\section{Objectives}

In the current study, the virus shedding in respiratory, blood, stool, and urine samples of infected patients was examined using quantitative real-time PCR (qRT-PCR) assay. 


\section{Methods}

\subsection{Study Setting and Design}

The oropharyngeal/nasopharyngeal swab specimens, in viral transport media (VTM), from suspected patients hospitalized at the hospitals of Markazi province were referred to the specialized virology laboratory of Imam Reza clinic (Arak University of Medical Sciences, Arak, Iran). A total number of 50 patients with high (Cq [cycle of quantification]-value $<22, \mathrm{n}=30$ ), moderate (Cq-value 22$32, \mathrm{n}=10$ ), and low (Cq-value 35-39, $\mathrm{n}=10$ ), virus titer in their respiratory specimens were enrolled in the current study (20 February to 4 March). There were 21 (42\%) females and 29 (58\%) males, with a mean age of 46 years (range, 26 to 81 years). Since no quantitative RT-qPCR assay has been used for the determination of SARS-CoV-2 viral load, patients were selected based on the Cq-values of qRT-PCR assay for the $\mathrm{E}$ gene of the virus. The day after reporting laboratory results, another oropharyngeal/nasopharyngeal swab sample along with $3 \mathrm{ml}$ blood, $5 \mathrm{ml}$ urine, and rectal swab sample (in VTM) of the sample patients were requested to be sent to the Virology laboratory and subjected to RNA extraction and qRT-PCR assay. Written informed consent was obtained from each patient prior to enrollment. The data of patients of the current study were not been neither reported nor will be submitted for any other publication.

\subsection{RNA Extraction and Qualitative Real-time PCR}

Viral RNA was extracted using QIAamp DSP Virus Kit (Qiagen, Hilden, Germany) in QIAcube extractor machines (Qiagen), based on the standard protocol of the manufacturer. Quantitative Real-time PCR (qRT-PCR) assay was performed by LightMix SarbecoV E-Gene plus EAV control (TIB MOLBIOL, Berlin, Germany) using QuantiNova Pathogen +IC master mix (Qiagen) in LightCycler ${ }^{\circledR} 96$ instruments (Roche Diagnostics, Mannheim, Germany). The oligonucleotide primer-probes are based on the hydrolysis probe technology that are designed for the E-gene (firstline screening assay) and RdRp-gene (confirmatory assay) (6). Reaction was set up in a $20-\mu \mathrm{l}$ volume containing $5 \mu \mathrm{l}$ master mix, 0.6 M of each primer, 0.3 M of each specific probe, and $5 \mu \mathrm{l}$ of extracted RNA. The amplification profile consisted of reverse-transcription at $50{ }^{\circ} \mathrm{C}$ for $10 \mathrm{~min}$, a single cycle of enzyme activation at $95^{\circ} \mathrm{C}$ for $2 \mathrm{~min}$ followed by 45 cycles of denaturation at $95{ }^{\circ} \mathrm{C}$ for 10 s and $60{ }^{\circ} \mathrm{C}$ for 30s with a single fluorescence acquisition. To ensure the quality of specimen collection and the absence of PCR inhibitors, the amplification of the human RnaseP gene was used as internal control in separate tubes and based on the above-mentioned reaction conditions.

\subsection{Statistical Analysis}

The difference between Cq-values of two successive respiratory specimens and the internal control gene in different samples was compared using the paired-sample ttest and One-way ANOVA, respectively. P values of less than 0.05 were considered statistically significant. All statistical analyses were calculated using SPSS software (version 16; SSPS Inc., 184 Chicago, IL, USA).

\section{Results}

The results of RT-qPCR assay indicate that SARSA-CoV-2 was detectable in all respiratory specimens and no positive results were observed in the urine samples of all patients. The viral genome was diagnosed in three blood samples (5\%) and two rectal swabs (3.3\%). The Cq-values of positive results on the stool and blood samples of positive individuals were higher than $4 \mathrm{Cq}$ above the value of the respiratory specimen of the same patient. No patients showed both positive blood and stool sample at the same time.

The Cq-value of the internal control gene was used as an indicator for specimen quality, and the lack of inhibitors in the amplification reaction was arbitrarily defined to be $<26$. There was not a significant difference in the Cq-values of two successive respiratory specimens of the patients $(\mathrm{P}=0.465$ by paired-sample $\mathrm{t}$-test). The mean Cq-values of the internal control gene was higher in the blood samples; nevertheless, the difference was not statistically significant (one-way ANOVA). To evaluate the accuracy of the negative results, all rectal swabs, blood, and urine specimens were reassessed using the 2019-nCoV Nucleic Acid Diagnostic kit (Sansure biotech, Changsha, China), and the results remained negative.

Within a few weeks of the emergence of SARS-CoV-2 and the COVID-19, controversy exists about the routes of viral spread and its existence in different biological samples. Understanding the routes of the virus shedding is essential in terms of defining biosafety for health care workers and society and managing patients in hospitals or home isolation. The probable existence of SARS-CoV-2 in different biological samples has been evaluated in a few studies (7-9). In this regard, some reports declare that the virus is detected in the blood sample (7), the others could not diagnose it in the blood (9). To the best of our knowledge, no study has reported the detection of SARS-CoV-2 in the urine sample. On 
the other hand, the viral RNA is reported in the fecal sample of more than $30 \%$ of patients (7).

Our data confirm the lack of viral genome in the urine. The result is discordant with the study conducted by Xie et al. that the virus is not detected in the blood of infected patients (9). Additionally, the viral RNA was detected in the 3.3\% rectal swab of infected patients. In the current study, we categorize the study population based on the virus titer in their respiratory specimen, to try to cover patients with different stages of the disease. The major group consisted of the patients whose virus titer in their upper-respiratory secretion was high, assuming that this group experienced the acute onset of the disease.

The virus in the upper-respiratory mucosa of this group was high, and hence, the virus could have been detected if it had existed in the blood or stool. Patients with moderate or low titer of the virus were either in the late phase of the disease or the very beginning of the viral infection, and there was a possibility that in these stages, the patients might have been viremic. The other advantage of the current study was to evaluate the internal control gene in all analyzed specimens in order to prevent false-negative results due to the poor sample collection.

\section{Discussion}

In the current study, the virus is detected in $5 \%$ of individuals, 2 with high and 1 with moderate virus titer. The percentage of a positive result in the current study is comparable to the data obtained by Wang et al. (10). On the other hand, the discordant percentage of positive rectal swab between this and previous studies might be due to the sample size, type and quality of specimens, and genetic variations between the two populations. In the current study, a rectal swab was used, but in a few studies, stool samples were examined directly. Additionally, none of the patients in our study had diarrhea, while in some reports, diarrheal samples were also examined (9).

Some studies that examined pediatric samples reported that the age and physiological differences between different age groups might be considered contributing factors. Finally, the genetic variations between the population in China and Iran may be a factor that should not be easily neglected. Despite the difference in the percentage of positive stools samples in different studies and the fact that only a few patients have positive results, the positive qRTPCR in stool does not necessarily reflect the active replication of the virus in the gastrointestinal tract, and it seems that the residual viral RNA from a digested virus in the digestive system provides a template for qRT-PCR.

\subsection{Conclusions}

In conclusion, the results of the current study showed that SARS-CoV-2 is mainly detected in the respiratory samples. The virus is not detectable in the urine and only a few blood and stool. The importance of viremia in virus spread in the human population through transfusion needs further investigation.

\section{Acknowledgments}

The authors thank Elham Taheri, Somayeh Salimi, and Masoumeh Jamshidi for their assistance in this research.

\section{Footnotes}

Authors' Contribution: MM and BK participated in the design of the study, ER drafted the manuscript, HS revised the manuscript critically for important intellectual content, and MJ and BK managed the project implementation of the study. All authors read and approved the final version of the manuscript.

Conflict of Interests: The authors have no conflicts of interest.

Ethical Approval: The study was approved by the Ethics Committee of Arak University of Medical Sciences, in accordance with the declaration of Helsinki (ethics number IR.ARAKMU.REC.1398.340).

Funding/Support: This study was supported by a grant from the Deputy for research and technology, Arak University of Medical Sciences. The funders had no role in study design, data collection, and analysis, decision to publish, or preparation of the manuscript.

Informed Consent: Written informed consent was obtained from each patient prior to enrollment.

\section{References}

1. Zhu N, Zhang D, Wang W, Li X, Yang B, Song J, et al. A Novel coronavirus from patients with pneumonia China, 2019. $N$ Engl J Med. 2020;382(8):727-33. doi: 10.1056/NEJMoa2001017. [PubMed: 31978945]. [PubMed Central: PMC7092803].

2. World Health Organization. Coronavirus disease (COVID-2019) situation reports. 2020. 2020. Available from: https://www.who.int/ emergencies/diseases/novel-coronavirus-2019/situation-reports. 
3. Wang D, Hu B, Hu C, Zhu F, Liu X, Zhang J, et al. Clinical characteristics of 138 hospitalized patients with 2019 novel coronavirusinfected pneumonia in Wuhan, China. JAMA. 2020;323(11):1061-9. doi: 10.1001/jama.2020.1585. [PubMed: 32031570]. [PubMed Central: PMC7042881].

4. Zhou P, Yang XL, Wang XG, Hu B, Zhang L, Zhang W, et al. A pneumonia outbreak associated with a new coronavirus of probable bat origin. Nature. 2020;579(7798):270-3. doi: 10.1038/s41586-020-2012-7. [PubMed: 32015507]. [PubMed Central: PMC7095418].

5. Dong E, Du H, Gardner L. An interactive web-based dashboard to track COVID-19 in real time. The Lancet Infectious Diseases. 2020;20(5):533-4. doi:10.1016/s1473-3099(20)30120-1.

6. Corman VM, Landt O, Kaiser M, Molenkamp R, Meijer A, Chu DK, et al. Detection of 2019 novel coronavirus (2019-nCoV) by real-time RT-PCR. Euro Surveill. 2020;25(3). doi:10.2807/1560-7917.ES.2020.25.3.2000045. [PubMed: 31992387]. [PubMed Central: PMC6988269].

7. Zhang J, Wang S, Xue Y. Fecal specimen diagnosis 2019 novel coronavirus-infected pneumonia. J Med Virol. 2020;92(6):680-2. doi: 10.1002/jmv.25742. [PubMed: 32124995]. [PubMed Central: PMC7228355].

8. Zhang W, Du RH, Li B, Zheng XS, Yang XL, Hu B, et al. Molecular and serological investigation of 2019-nCoV infected patients: implication of multiple shedding routes. Emerg Microbes Infect. 2020;9(1):3869. doi: 10.1080/22221751.2020.1729071. [PubMed: 32065057]. [PubMed Central: PMC7048229].

9. Xie C, Jiang L, Huang G, Pu H, Gong B, Lin H, et al. Comparison of different samples for 2019 novel coronavirus detection by nucleic acid amplification tests. Int J Infect Dis. 2020;93:264-7. doi: 10.1016/j.ijid.2020.02.050. [PubMed: 32114193]. [PubMed Central: PMC7129110].

10. Wang W, Xu Y, Gao R, Lu R, Han K, Wu G, et al. Detection of SARS-CoV2 in different types of clinical specimens. JAMA. 2020;323(18):1843-4. doi: 10.1001/jama.2020.3786. [PubMed: 32159775]. [PubMed Central: PMC7066521]. 